\title{
Notfalldienst: Alle wollen ihn, aber wer soll inn bezahlen?
}

\section{Remo Osterwalder}

Dr. med., Vizepräsident der FMH, Departementsverantwortlicher Rettungswesen

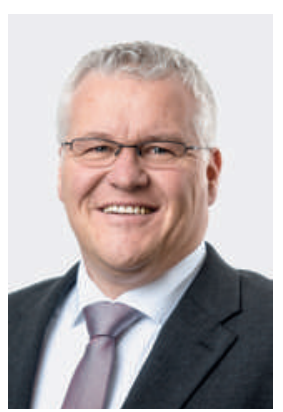

Wer kennt es nicht: Nach dem langen Gang durch die Administration bis zur Eröffnung der eigenen Praxis wird man durch die Kollegen der Ärztegesellschaft nicht nur über die Rechte aufgeklärt, sondern auch über die Pflichten. Anders gesagt, man wird mit Freuden in den Notfalldienst aufgenommen, was in den meisten Regionen nach wie vor eine Pflicht ist. Eine Pflicht, welche wir schon in der Facharzt-Ausbildung mit den Notfalldiensten im Spital wahrnehmen, aber auch eine, welche nebst Freud auch die Schattenseiten dieser Leistung im Gesundheitswesen aufzeigt. Am Anfang einer selbständigen Karriere sind diese Dienste noch attraktiv, da der eine oder andere Patient noch einen Hausarzt sucht und auf diese Weise auch findet. Die Realität ist aber vielmals so, dass diese Notfall-Konsultationen leider zum Teil unbezahlt bleiben. Einzelne Patienten haben das Gefühl, dass dieses Angebot vom Staat zur Verfügung gestellt wird, oder haben generell Schwierigkeiten, dieser administrativen Angelegenheit nachzukommen.

\section{Der Notfalldienst ist eine wichtige Leistung im Interesse der Bevölkerung.}

So heterogen wie die Zusammensetzung der Schweiz ist auch die Organisation des Notfalldienstes. Heute gibt es bereits Regionen, in welchen der Notfalldienst mittels der vor Ort tätigen Grundversorgerinnen und -versorger nicht mehr garantiert werden kann, insbesondere wenn diese das Rentenalter erreichen. Gewisse kritische Situationen können mittels einer Kooperation mit einem Grundversorger-Spital abgefedert werden. Das Hauptproblem dieser Institutionen ist, dass sie nicht sehr mobil sind und deshalb oft keine Behandlungen vor Ort, beispielsweise in Form von Hausbesuchen, anbieten können. Dies ist vor allem in ländlichen Regionen der Fall. Allgemein stellt sich die Frage, wie die Attraktivität solcher Notfall-Einsätze für junge Kolleginnen und Kollegen gesteigert werden könnte. Denn man darf nicht einfach glauben, dass es zum Homo medicus gehört, aus Freude an der Arbeit ganze Wochenenden getrennt von seiner Familie zu verbringen. Nicht nur die Erwartungen der nachfolgenden Generationen junger Ärztinnen und Ärzte ändern sich, sondern auch die berechtigten Ansprüche ihrer Familien.

Grundsätzlich sind die Kantone für den Schutz und die Förderung der Gesundheit ihrer Bevölkerung verantwortlich, inklusive Notfall. Dies wird in beschränktem Masse durch deren staatliche Institutionen sichergestellt - beschränkt deshalb, weil die Hausbesuche

\section{Notfall-Leistungen sollten auch ambulant} in Form von Vorhalteleistungen entgolten werden.

oder wohnortnahe Konsultationen eher selten dazugehören. Im Gegensatz zu den Spitälern, in welchen Notfall-Leistungen mit Hilfe der von der Allgemeinheit finanzierten Vorhalteleistungen (Kantonsbeiträge) entgolten werden, ist dies für die niedergelassenen Kollegen eher selten. Aus Sicht der Kantone müsste hier ein Interesse bestehen, solche Notfall-Leistungen ebenfalls in Form von Vorhalteleistungen abzugelten. Ebenso wie die gesetzliche Pflicht zur Teilnahme an Notfalldiensten in allen 26 Kantonen besteht, könnte diese Massnahme die Grundlage zur Entschädigung und Aufwertung dieses Dienstes zum Vorteil der Bevölkerung bilden. Die Ärztinnen und Ärzte ihrerseits haben vorgespurt und den Notfalldienst bereits aufgewertet, zum Beispiel durch das Angebot des Dienstarztkurses der Schweizerischen Gesellschaft für Notfall- und Rettungsmedizin (SGNOR) oder durch das neue notfallmedizinische Curriculum für die Medizinstudierenden der Universität Basel in Zusammenarbeit mit der SGNOR und der FMH. Qualität steht für die Ärzteschaft an erster Stelle, aber eine proaktive Attraktivitätssteigerung und Anerkennung ist dringend nötig - und zwar bevor grossflächig eine Lücke in der Versorgung des Notfalldienstes entsteht. 